\title{
Advancements in time-resolved $x$-ray laser induced time-of-flight photoelectron spectroscopy
}

\author{
A. J. Nelson, J. Dunn, K. Widmann, T. Ao, Y. \\ Ping, J. Hunter, A. Ng
}

July 29,2005

SPIE 50th International Symposium on Optical Science \& Technology

San Diego, CA, United States

July 31, 2005 through August 4, 2005 
This document was prepared as an account of work sponsored by an agency of the United States Government. Neither the United States Government nor the University of California nor any of their employees, makes any warranty, express or implied, or assumes any legal liability or responsibility for the accuracy, completeness, or usefulness of any information, apparatus, product, or process disclosed, or represents that its use would not infringe privately owned rights. Reference herein to any specific commercial product, process, or service by trade name, trademark, manufacturer, or otherwise, does not necessarily constitute or imply its endorsement, recommendation, or favoring by the United States Government or the University of California. The views and opinions of authors expressed herein do not necessarily state or reflect those of the United States Government or the University of California, and shall not be used for advertising or product endorsement purposes. 


\title{
Advancements in time-resolved x-ray laser induced time-of-flight photoelectron spectroscopy
}

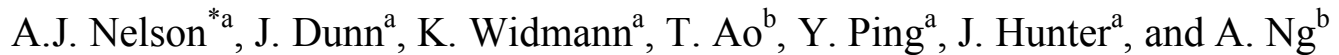 \\ ${ }^{a}$ Lawrence Livermore National Laboratory, Livermore, CA 94551 \\ ${ }^{b}$ Dept. of Physics, University of British Columbia, Vancouver 89154
}

\begin{abstract}
Time-resolved soft x-ray photoelectron spectroscopy is used to probe the non-steady-state evolution of the valence band electronic structure of laser heated ultra-thin $(50 \mathrm{~nm})$ metal foils and bulk semiconductors. Single-shot soft x-ray laser induced time-of-flight photoelectron spectroscopy with picosecond time resolution was used in combination with optical measurements of the disassembly dynamics that have shown the existence of a metastable liquid phase in $f s$-laser heated metal foils persisting 4-5 ps. This metastable phase is studied using a $527 \mathrm{~nm}$ wavelength 400 fs laser pulse containing $0.3-2.5 \mathrm{~mJ}$ laser energy focused in a large $500 \times 700 \mu \mathrm{m}^{2}$ spot to create heated conditions of $0.2-1.8 \mathrm{x}$ $10^{12} \mathrm{~W} \mathrm{~cm}^{-2}$ intensity. The unique LLNL COMET compact tabletop soft $\mathrm{x}$-ray laser source provided the necessary high photon flux, highly monoenergetic, picosecond pulse duration, and coherence for observing the evolution of changes in the valence band electronic structure of laser heated metals and semiconductors with picosecond time resolution. This work demonstrates the continuing development of a powerful new technique for probing reaction dynamics and changes of local order on surfaces on their fundamental timescales including phenomena such as non-thermal melting, chemical bond formation, intermediate reaction steps, and the existence of transient reaction products.
\end{abstract}

Keywords: time-of-flight photoemission, x-ray laser

\section{INTRODUCTION}

The application of compact high efficiency collisional excitation x-ray lasers operating with higher repetition rate pumps [1-4] for probing dynamical changes of matter with ultrafast time resolution has come of age. At Lawrence Livermore National Laboratory (LLNL) we have been developing time-of-flight techniques for photoelectron spectroscopy induced by single-shot $\mathrm{x}$-ray laser pulses in a pump-probe investigation of changes in the electronic structure of materials undergoing ultrafast laser heating $[5,6]$.

Time-of-flight (ToF) photoelectron spectroscopy (PES) with a pulsed synchrotron radiation source was first investigated as a means to determine energy and angular distributions in low photon energy regimes. [7] Other researchers have used ToF to observe static chemical shifts in the $\mathrm{Si} 2 p$ core level electronic structure for $\mathrm{SiO}_{2}$ and $\mathrm{Si}_{3} \mathrm{~N}_{4}$ samples using $2.5 \mathrm{~ns}$ laser produced plasma (LPP), $255 \mathrm{eV}$ x-ray pulses after integrating for $\sim 100$ shots [8,9]. These studies while successful have several significant limitations which rule out a pump-probe experiment to study dynamic processes in a material induced by shocks, e.g. melting, phase changes. Specifically, the low photon fluxes $\sim 2.5 \times 10^{8}$ photons/pulse on the sample required multiple shots to record a photoelectron spectrum. In addition, low x-ray source energy resolution, $\Delta \mathrm{E} / \mathrm{E} \sim 1.4 \times 10^{-2}$, and long $2.5 \mathrm{~ns}$ pulse duration limited the energy resolution of the photoelectron spectrum and therefore the ability to distinguish fine details in the electronic structure. Finally, time resolved observation of changes in the electronic structure during dynamic processes requires pulse durations on the order of a picosecond.

Higher order harmonic $(\mathrm{HOH})$ sources overcome the limitations of the LPP sources and have the advantage of shorter pulse duration, e.g. less than $60 \mathrm{fs}$, at a high repetition rate [10-12] which makes them very attractive for dynamic pump-probe experiments. However, the presence of multiple harmonics requires further wavelength selection with multilayers or diffraction gratings. In addition, the lower photon fluence/shot requires many shots to achieve good signal statistics. Thus the need for monochromatic, high photon fluence, short pulse x-ray source for performing time-resolved dynamic studies.

Time-resolved core-level photoelectron spectroscopy performed at the Advanced Light Source has probed both the local arrangement of atoms (providing spatial information) as well as the bonding character of the valence electrons 
(providing electronic information). [13] In these experiments, the total elapsed time from laser heating to final ejected particle formation was less than $100 \mathrm{ps}$ and the results indicated that in the intermediate phase there is a metallic fluid where the electrons become mobile. The mobile electrons efficiently screen the x-ray core-hole leading to a lowering of the binding energy. The spectral modifications in this phase are due to the fact that core-level photoelectron spectroscopy is responsive to the character of the valence electronic wavefunction.

Each of the aforementioned studies with the different sources has their associated strengths and weaknesses. We will show that the LLNL COMET (Compact Multipulse Terawatt) x-ray laser, which is highly monochromatic with a combination of high photon fluence and ps pulse duration, is ideal to perform dynamic optical pump-x-ray probe electronic structure measurements on a single-shot. This paper presents results that demonstrate this capability.

\section{EXPERIMENTAL}

The experiments were performed on the COMET laser system at LLNL [2]. This laser, operating at $1054 \mathrm{~nm}$ wavelength, utilizes the technique of chirped pulse amplification to produce two high power beams at a rate of 1 shot every 4 minutes. A short pulse nominally 500 fs to 25 ps and a long 600 ps (FWHM) pulse is focused in a high intensity line focus with a traveling wave geometry to generate the Ni-like Pd ion $4 d-4 p$ x-ray laser line at $14.7 \mathrm{~nm}(84.5 \mathrm{eV})$. Total energy in the two beams is of order $3-7 \mathrm{~J}$ to produce lasing where the peak-to-peak delay between the laser pulses is found to be optimal at $700 \mathrm{ps}$ with the short pulse arriving after the long pulse. High photon flux/shot, high monochromaticity, and short pulse duration when combined with small source area and beam divergence properties of the $14.7 \mathrm{~nm}$ line [14] give ultra-high peak brightness $\sim 10^{25} \mathrm{ph} . \mathrm{mm}^{-2} \mathrm{mrad}^{-2} \mathrm{~s}^{-1}(0.1 \% \mathrm{BW})^{-1}$ assuming a 2 ps pulse duration. Overall, the $14.7 \mathrm{~nm}$ peak brightness is $5-6$ orders of magnitude higher than $3^{\text {rd }}$ generation synchrotron undulator sources. However, third generation synchrotron undulator sources still have higher average brightness of $0.5-$ $6 \times 10^{18} \mathrm{ph} . \mathrm{mm}^{-2} \mathrm{mrad}^{-2} \mathrm{~s}^{-1}(0.1 \% \mathrm{BW})^{-1}$ at $50-10 \mathrm{~nm}$, respectively. The technique of electron time-of-flight (e-ToF) spectroscopy requires a monochromatic, ps pulsed source for efficient $\mathrm{x}$-ray laser induced photoelectron spectroscopy (PES).

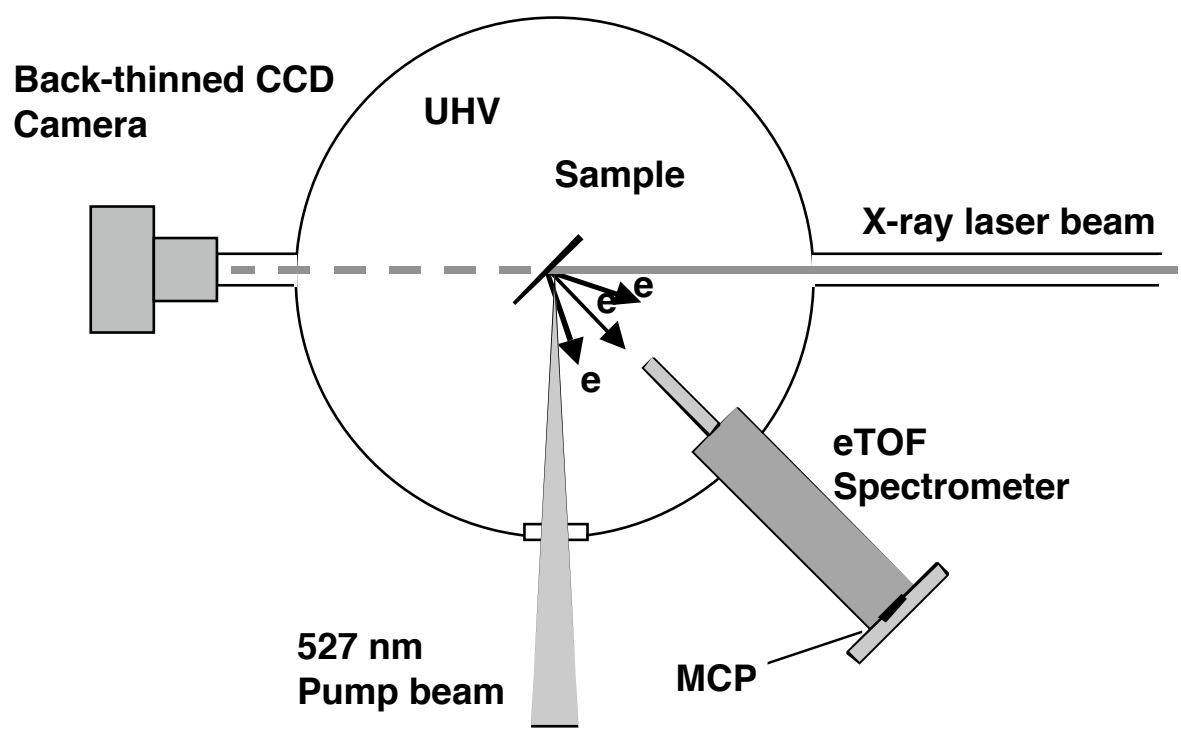

Figure 1. Schematic for the optical pump - x-ray laser probe of material surfaces.

Figure 1 shows a schematic of the x-ray laser photoelectron spectroscopy experimental setup. A fast 400 fs (FWHM), $527 \mathrm{~nm}$ wavelength laser pump beam can be focused onto the sample to produce rapid heating and induce phase changes. The x-ray laser probe is collimated by a normal incidence Mo:Si multilayer spherical mirror and relayed along the beamline by a $45^{\circ} \mathrm{Mo}: \mathrm{Si}$ multilayer flat mirror. The narrow reflectivity window of the mirrors selects the $\mathrm{x}$-ray laser wavelength and minimizes other plasma x-rays reaching the sample. A pinhole or thin filter isolates the beamline from the experimental ultra-high vacuum (UHV) chamber, operating below $10^{-8}$ mbar pressure. The x-ray laser probes the 
material surface being heated by the optical laser pump, at various times and induces photoemission. These low energy photoelectrons, with K.E. $<84.5 \mathrm{eV}$, emitted from the valence band and shallow core levels are detected by the e-ToF spectrometer, the operation of which is described in detail elsewhere. [15] The signal is acquired and digitized using a fast $6 \mathrm{GHz}$ oscilloscope.

\section{RESULTS AND DISCUSSION}

Figure 2 presents the static x-ray laser induced photoemission e-ToF photoemission spectra for the Ge (100) crystal illuminated with $10^{8}-10^{9} \mathrm{x}$-ray laser photons. Space charge effects were not evident at these intensities. Each spectrum represents the average sum of two to four shots and the retard (or drift) potentials were varied as shown on each spectrum to improve time resolution and consequently energy resolution. The x-ray prompt peak seen in the spectrum with no drift voltage is generated from scattered $\mathrm{x}$-rays off the Ge surface hitting the micro-channel plate (MCP) detector a distance of $\mathrm{L}=43.75 \mathrm{~cm}$ away. [15] The $\mathrm{x}$-ray prompt peak occurs $\mathrm{L} / \mathrm{c}=1.46 \mathrm{~ns}$ after the $\mathrm{x}$-ray laser hits the sample, where $\mathrm{c}$ is the speed of light, and can be used as a timing fiducial. These spectra are more indicative of a Geoxide layer in the absence of sputter ion cleaning due to the $\sim 1 \mathrm{~nm}$ mean escape depth of the photoemitted electrons.

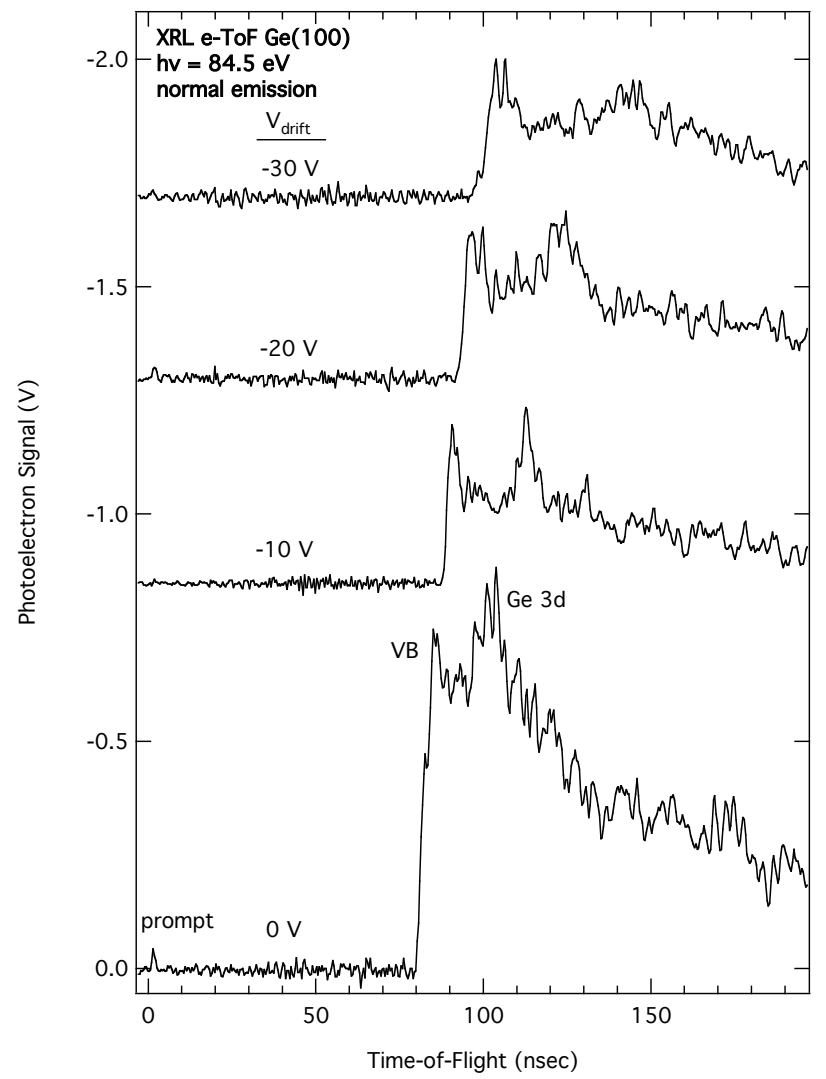

Figure 2. X-ray laser induced photoelectron spectra for Ge(100) surface using various drift voltages.

The upper valence band of oxidized Ge is composed primarily of Ge $4 p$ occupied states that hybridize with $\mathrm{O}$ $2 p$ states. [16] The work function for Ge (100) is $4.8 \mathrm{eV}$ and thus the kinetic energy for these valence band photoelectrons, the first e-ToF event, will be $\sim 79.7 \mathrm{eV}$. The kinetic energies of the Ge $3 d_{5 / 2,3 / 2}$ spin-orbit pair are 50.5 $\mathrm{eV}$ and $50.0 \mathrm{eV}$, respectively, and are the next expected event in the spectrum. In addition, the photoionization crosssections for the Ge $4 p$ valence band and the Ge $3 d$ shell at an excitation energy of $80 \mathrm{eV}$ are $0.039 \mathrm{Mb}$ and $6.999 \mathrm{Mb}$, respectively [17] which would imply that the strongest peak should be the Ge $3 d$ core. However, oxidized Ge has an $\mathrm{O}$ $2 p$ component in the valence band with a photoionization cross-section of $2.064 \mathrm{Mb}$ at an excitation energy of $80 \mathrm{eV}$. Therefore, based on the observed peak height ratios for valence band and Ge $3 d$ core-level emission, the spectra are indeed indicative of an oxidized surface layer. Non-equilibrium occupation of the valence band states created by the $\mathrm{x}$ - 
ray laser intensity $\left(10^{6} \mathrm{~W} / \mathrm{cm}^{2}\right)$ may also contribute to the unusual electron energy distribution at the valence band edge. The broad background under the Ge $3 d$ peak originates from secondary electron emission that represents elastically and multiply scattered electrons.

As we increase the negative drift voltage in the flight tube of the e-ToF analyzer and thus reduce the kinetic energy of the photoemitted electrons, the valence band threshold and separation between the valence band and core-level events change (Fig. 2). The notable decrease in photoelectron signal for the $-30 \mathrm{~V}$ retarding field is due to a decrease in the effective solid angle of photoelectrons reaching the MCP detector. [18] In the case of no drift voltage, the photoelectron yield as given by $\mathrm{N}_{\mathrm{p}}=$ Fon $\lambda \mathrm{T}$ can be estimated from the $\mathrm{x}$-ray fluence on the sample after filter and mirror losses $(\mathrm{F}=$ $10^{9}$ photons/pulse), the Ge $3 d$ photoionization cross-section $\left(\sigma \approx 7.0 \times 10^{-18} \mathrm{~cm}^{2}\right)$, the Ge number density $\left(\mathrm{n}=4.4 \times 10^{22}\right.$ $\left.\mathrm{cm}^{-3}\right)$, the escape depth $\left(\lambda=5 \times 10^{-8} \mathrm{~cm}\right)$, and the angular collection efficiency of the MCP detector $\left(\mathrm{T}=2.5 \times 10^{-4}\right)$. Therefore, we can expect $3.9 \times 10^{3}$ photoelectrons/pulse to arrive at the MCP detector and produce signal. We observe single-shot photoelectron signals in the range of $200-400 \mathrm{mV}$ without retarding.

We now apply this demonstrated capability to the time-resolved examination of changes in the electronic structure of $\mathrm{Cu}$ undergoing ultrafast isochoric laser heating. The ultra-thin $\mathrm{Cu}$ target consists of $50 \mathrm{~nm} \mathrm{Cu}$ on a $20 \mathrm{~nm} \mathrm{C}$ support. X-ray photoelectron spectroscopy (XPS) was used to characterize the surface chemistry of the ultra-thin Cu foil prior to the dynamic pump-probe experiments. Figure 3(a) shows the survey spectrum and the high-resolution $\mathrm{Cu} 2 \mathrm{p}$ core-level spectrum for the as received surface. Carbon, oxygen, and nitrogen contamination are evident in the foil. The Si contamination is due to the mounting procedure.
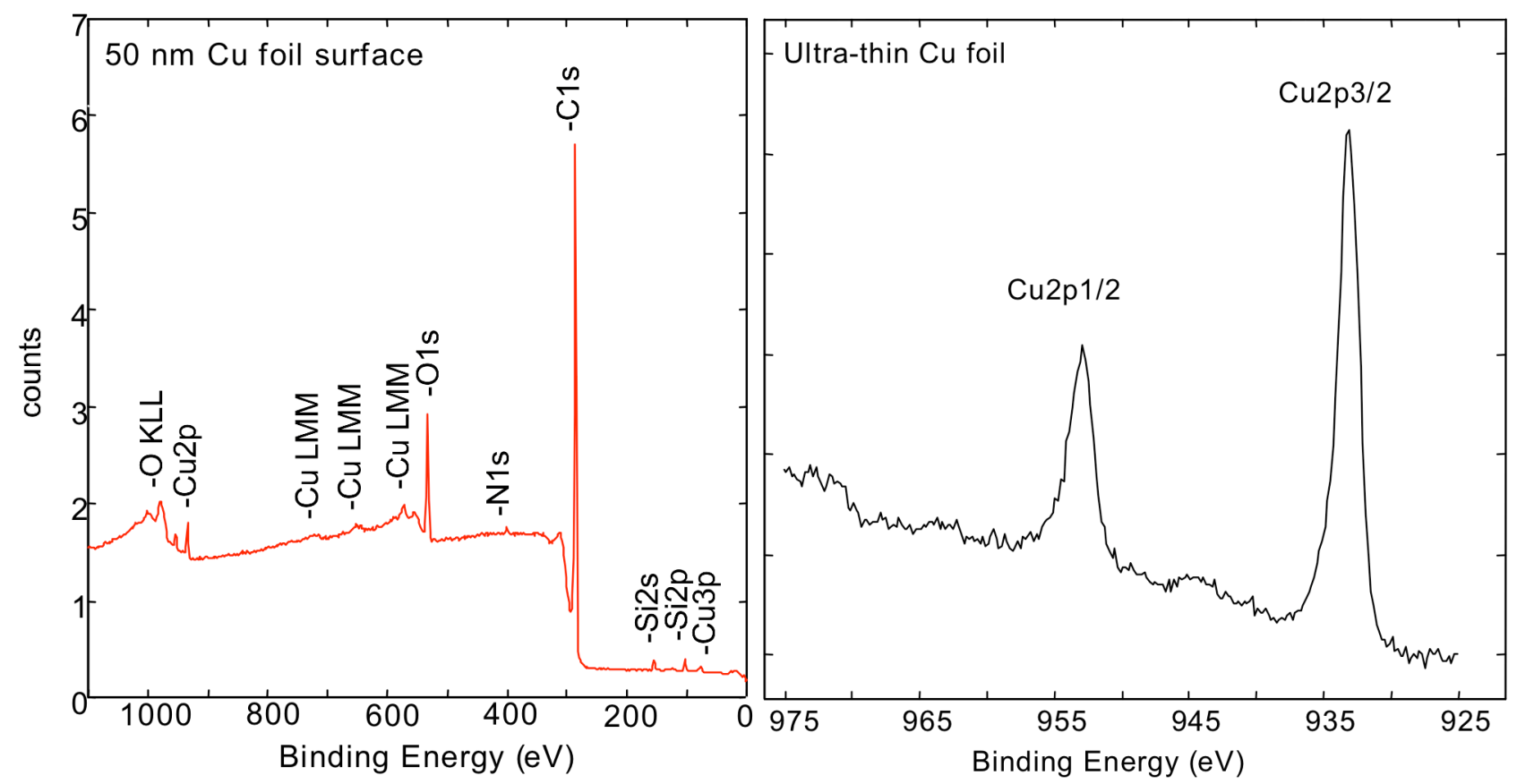

Figure 3. (a) XPS survey spectrum and (b) high-resolution $\mathrm{Cu} 2 \mathrm{p}$ core-level spectrum of ultra-thin $\mathrm{Cu}$ foil.

Examination of the $\mathrm{Cu} 2 p$ line-shape reveals no plasmon loss features indicative of $\mathrm{CuO}$. [19] However, the nanocrystalline $\mathrm{Cu}$ could possibly be preferentially oxidized at the grain boundaries and on the surface as $\mathrm{Cu}_{2} \mathrm{O}$. Nevertheless, these oxides were easily reduced using ultraviolet light prior and during $\mathrm{x}$-ray laser induced photoelectron spectroscopy. [20,21]

Figure 4 presents the single-shot e-ToF photoemission spectra of the $3 d$ valence band of static and laser heated ultra-thin polycrystalline $\mathrm{Cu}$ foil illuminated with $10^{8}-10^{9} \mathrm{x}$-ray laser photons. This sample is sufficiently thin to observe the transmitted x-ray laser beam simultaneously with the photoelectron signal. Again the $\mathrm{x}$-ray prompt peak can be used as 

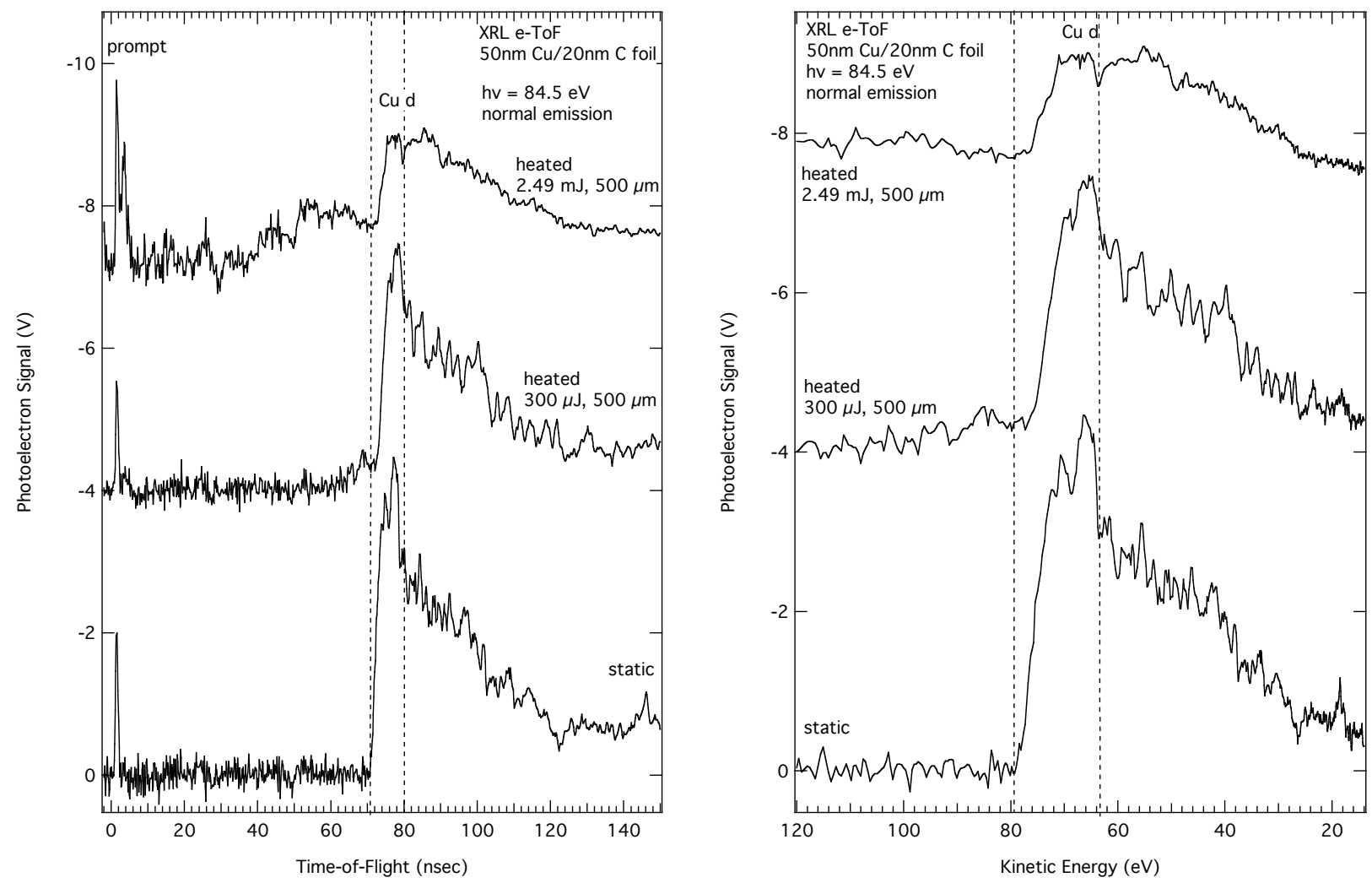

Figure 4. Single-shot x-ray laser induced photoelectron spectra for static and laser heated ultra-thin polycrystalline Cu foil (a) signal versus time-of-flight and (b) signal versus kinetic energy. Dashed lines represent limits of photoemission threshold and $\mathrm{Cu} d$ band emission.

a timing fiducial. This scattered signal intensity is also sensitive to the surface finish of the sample. The mean escape depth of the photoemitted electrons is on the order of $\sim 1 \mathrm{~nm}$ for this kinetic energy. In addition, the photoelectron yield as given by $\mathrm{N}_{\mathrm{p}}=\mathrm{Fon} \lambda \mathrm{T}$ can be estimated from the $\mathrm{x}$-ray fluence on the sample after filter and mirror losses $\left(\mathrm{F}=10^{9}\right.$ photons/pulse), the $\mathrm{Cu} 3 d$ photoionization cross-section $\left(\sigma \approx 8.7 \times 10^{-18} \mathrm{~cm}^{2}\right)$ [17], the $\mathrm{Cu}$ number density $(\mathrm{n}=8.4 \times$ $\left.10^{22} \mathrm{~cm}^{-3}\right)$, the escape depth $\left(\lambda=5 \times 10^{-8} \mathrm{~cm}\right)$, and the angular collection efficiency of the MCP detector $\left(\mathrm{T}=2.5 \times 10^{-}\right.$ $\left.{ }^{4}\right)$. Therefore, we can expect $9.1 \times 10^{3}$ photoelectrons/pulse to arrive at the MCP detector and produce signal. We observe photoelectron signals with amplitudes of 2-4 V with a single x-ray laser shot and without the application of a retarding field.

Under static conditions (lower spectrum Fig. 4) the $3 d$ valence band electrons will have the highest kinetic energies as governed by $\mathrm{E}_{\mathrm{k}}=\mathrm{h} v-\mathrm{E}_{\mathrm{b}}-\phi$, where $\mathrm{h} v$ is the energy of the incident photon $(84.5 \mathrm{eV}), \mathrm{E}_{\mathrm{b}}$ is the binding energy of the photoelectron relative to the Fermi level and $\phi$ is the work function of the material $(\phi=4.6 \mathrm{eV} \mathrm{for} \mathrm{Cu})$. Valence band photoemission will thus be the first and strongest event in the e-ToF spectrum. The strong $\mathrm{Cu} d$-state emission observed at the valence band maximum, i.e. first e-ToF event is indicative of a high density of states (filled $d$-states) that are $2 \mathrm{eV}$ below the Fermi level. [22] The strong emission corresponds to direct transitions from $d$-like occupied bands to unoccupied bands above the Fermi energy. The initial as well as the final states determine the appearance, position, and intensity of these structures, and strongly depend on the applied photon energy. Non-equilibrium perturbations of the valence band states created by the $\mathrm{x}$-ray laser irradiance $\left(10^{6} \mathrm{~W} / \mathrm{cm}^{2}\right)$ also contribute to the unusually abrupt electron energy distribution at the valence band edge. Displaying the ambient $\mathrm{x}$-ray laser induced $\mathrm{Cu}$ valence band photoemission spectrum versus kinetic energy (Figure 4(b) lower spectrum) yields an energy distribution curve that is qualitatively comparable to $\mathrm{Cu}$ valence band photoemission spectra presented in the literature [23] that were acquired using synchrotron radiation. 
UCRL-PROC-214158

\section{CONCLUSIONS}

We have successfully validated $\mathrm{x}$-ray laser induced time-of-flight photoemission by measuring the valence band and shallow core-level photoemission in room temperature bulk materials. We have also successfully demonstrated the optical pump-x-ray laser probe characterization capability to observe the evolution of changes in the electronic structure of laser heated ultra-thin metal foils with picosecond time resolution. Single-shot spectra have been achieved showing that the incident photon number is more than enough for these applications. We will further investigate and characterize the range of x-ray laser intensities on the sample and the effect of space charge on the nonequilibrium electron energy distribution in future studies.

\section{ACKNOWLEDGMENTS}

The authors would like to thank Mark Eckart and Al Osterheld for their continued support. This work was performed under the auspices of the US Department of Energy by the University of California Lawrence Livermore National Laboratory under Contract No. W-7405-Eng-48 and by US Department of Energy Grant No. DE-FG0398DP00208.

\section{REFERENCES}

1. B.R. Benware, C.H. Macchietto, C.H. Moreno, and J.J. Rocca, "Demonstration of a high average power tabletop xray laser", Phys. Rev. Lett. 81(26), 5804-5807 (1998).

2. J. Dunn, Y. Li, A. L. Osterheld, J. Nilsen, J. R. Hunter, and V. N. Shlyaptsev, "Gain Saturation Regime for LaserDriven Tabletop, Transient Ni-like Ion X-Ray Lasers”, Phys. Rev. Lett. 84, 4834-4837 (2000).

3. S. Sebban, R. Haroutunian, Ph. Balcou, G. Grillon, A. Rousse, S. Kazamias, T. Marin, J. P. Rousseau, L. Notebaert, M. Pittman, J. P. Chambaret, A. Antonetti, D. Hulin, D. Ros, A. Klisnick, A. Carillon, P. Jaeglé, G. Jamelot, and J. F. Wyart, "Saturated Amplification of a Collisionally pumped Optical-Field-Ionization Soft X-Ray Laser at 41.8 nm", Phys. Rev. Lett. 86, 3004-3007 (2001).

4. T. Ozaki, R.A. Ganeev, A. Ishizawa, T. Kanai, and H. Kuroda, "Highly Directive 18.9 nm Nickel-like Molybdenum X-Ray Laser Operating at 150 mJ Pump Energy”, Phys. Rev. Lett. 89, 253902-1 - 4 (2002).

5. J. Dunn, R.F. Smith, J. Nilsen, A.J. Nelson, T.W. Van Buuren, S.J. Moon, J.R. Hunter, J. Filevich, J.J. Rocca, M.C. Marconi, V.N. Shlyaptsev, “A picosecond $14.7 \mathrm{~nm}$ x-ray laser for probing matter undergoing rapid changes”, $X$-Ray Lasers 2002: 8th International Conference on X-ray Lasers, ed. J.J. Rocca et al, AIP Conference Proceedings No. 641, 481 (2002).

6. A.J. Nelson, J. Dunn, T. van Buuren, J. Hunter, R.F. Smith, O. Hemmers, and D.W. Lindle, "X-ray laser induced time-of-flight photoelectron spectroscopy", Soft X-Ray Lasers and Applications V, SPIE 48th International Symposium on Optical Science \& Technology, Proc. SPIE Int. Soc. Opt. Eng. 5197, 168 (2003).

7. R.Z. Bachrach, F.C. Brown and S.B.M. Hagström, "Photoelectron-spectroscopy by time-of-flight technique using synchrotron radiation", J. Vac. Sci. Technol. 12(1), 309 (1975).

8. H. Kondo, T. Tomie, and H. Shimizu, "Time of flight photoelectron spectroscopy with a laser-plasma x-ray source", Appl. Phys. Lett. 69, 182 (1996).

9. H. Kondo, T. Tomie, and H. Shimizu, "Observation of chemical shifts of Si $2 \mathrm{p}$ level by an x-ray photoelectron spectroscopy system with a laser-plasma x-ray source”, Appl. Phys. Lett. 72, 2688 (1998).

10. A. Rettenberger and R. Haight, "Multivalley Electron Population Dynamics on the Ge(111):As Surface", Phys. Rev. Lett. 76, 1912-1915 (1996).

11. M. Bauer, C. Lei, K. Read, R. Tobey, J. Gland, M. M. Murnane, and H. C. Kapteyn, "Direct Observation of Surface Chemistry Using Ultrafast Soft-X-Ray Pulses", Phys. Rev. Lett. 87, 025501-1 (2001).

12. T. Munakata, T. Masuda, N. Ueno, S. Sakaya, T. Sugiyama, N. Takehiro, Y. Sonoda, "Microspot photoemission spectrometer based on FS-VUV radiation", Surf. Sci. 532, 1140-1144 (2003).

13. T.E. Glover, G. D. Ackerman, A. Belkacem, P. A. Heimann, Z. Hussain, R.W. Lee, H. A. Padmore, C. Ray, R.W. Schoenlein, W. F. Steele, and D. A. Young, "Metal-Insulator Transitions in an Expanding Metallic Fluid: Particle Formation Kinetics”, Phys. Rev. Lett. 90, 236102 (2003). 
14. J. Dunn, R.F. Smith, J. Nilsen, J.R. Hunter, T.W. Barbee, Jr., V.N. Shlyaptsev, J. Filevich, J.J. Rocca, M.C. Marconi, H. Fiedorowicz, and A. Bartnik, "Recent x-ray laser experiments on the COMET facility" SPIE Proc. 4505, 62 (2001).

15. O. Hemmers, S.B. Whitfield, P. Glans, H. Wang, D.W. Lindle, R. Wehlitz, and, I.A. Sellin, "High-resolution electron time-of-flight apparatus for the soft x-ray region" Rev. Sci. Instrum. 69, 3809-3807 (1998).

16. A. Goldoni, A. Santoni, M. Sancrotti, V.R. Dhanak and S. Modesti, "Photoemission and photoabsorption study of the high-temperature phases of the Ge(111) surface", Surf. Sci. 382, 336 (1997).

17. J.-J. Yeh and I. Lindau, "Atomic Subshell Photoionization Cross Sections and Asymmetry Parameters: $1 \leq Z \leq$ 103", Atomic Data and Nuclear Data Tables 32, 11 (1985).

18. A.J. Nelson, J. Dunn, T. van Buuren and J. Hunter, "X-ray laser induced photoelectron spectroscopy for single-state measurements", Appl. Phys. Lett. 85(25), 6290 (2004).

19. P.D. Kirsch and J.G. Ekerdt, "Chemical and thermal reduction of thin films of copper (II) oxide and copper (I) oxide", J. Appl. Phys. 90, 4256 (2001).

20. T.H. Fleisch, G.W. Zajac and J.O. Schreiner, "An XPS study of the UV photoreduction of transition and noblemetal oxides", Appl. Surf. Sci. 26, 488 (1986).

21. T.H. Fleisch G.J. Mains, "Reduction of copper oxides by UV-radiation and atomic-hydrogen studied by XPS", Appl. Surf. Sci. 10, 51 (1982).

22. N. Smith, "Photoelectron Energy Spectra and the Band Structures of the Noble Metals", Phys. Rev. B3, 1862 (1971).

23. J. Stöhr, F.R. McFeely, G. Apai, P.S. Wehner and D.A. Shirley, "Photoemission from Cu valence bands using 50175-eV synchrotron radiation", Phys. Rev. B14, 4431 (1976). 